\title{
Comunidades Profesionales de Aprendizaje en un contexto remoto por la emergencia sanitaria
}

\section{Professional Learning Communities in a remote context due to the health emergency}

Recibido: 15 de noviembre 2020, evaluado: 11 de enero 2021, aceptado: 5 de abril 2021

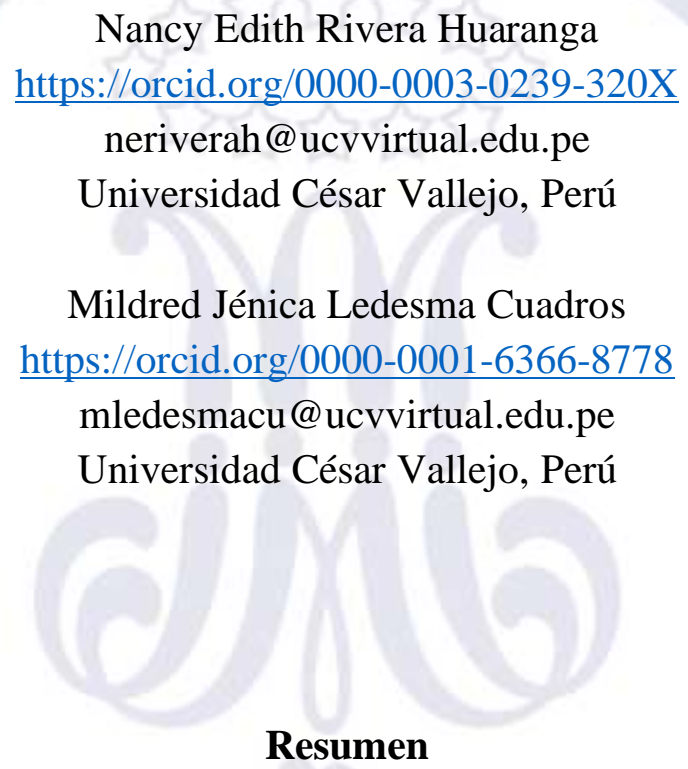

El estudio tuvo como objetivo determinar las diferencias que existen en los factores que describen las Comunidades Profesionales de Aprendizaje en las Instituciones Educativas de Lima Este en un contexto de trabajo remoto por la emergencia sanitaria, según nivel educativo, sexo, edad y formación académica. Se trabajó desde un enfoque cuantitativo y diseño descriptivo comparativo. La muestra de estudio estuvo constituida por 144 docentes de Lima Este, a quienes se le aplicó el cuestionario adaptado y validado por Bolívar (2017): Evaluación de las Comunidades Profesionales de Aprendizaje, en forma virtual. El análisis realizado con base en los datos estadísticos permitió establecer las diferencias a nivel de las dimensiones de las Comunidades Profesionales de Aprendizaje; la investigación evidencia que los factores que involucran las Comunidades Profesionales de Aprendizaje en las Instituciones Educativas de Lima Este, prevalecen un nivel de regular superior al 50\%, en las dimensiones de liderazgo compartido y de apoyo, visión y valores compartidos, aprendizaje colectivo y aplicación, práctica pro- 
fesional compartida, condiciones de apoyo: relaciones y condiciones de apoyo: estructura; seguido del nivel eficiente; igualmente, en todas las dimensiones superiores al $27.1 \%$; por lo que se puede afirmar que las comunidades profesionales en estudio vienen gestionándose adecuadamente por parte de los propios actores en estudio.

Palabras clave: comunidades profesionales de aprendizaje, contexto remoto, emergencia

\section{Summary}

The objective of the study was to determine the differences that exist in the factors that describe the Professional Learning Communities in the Educational Institutions of East Lima in a context of remote work due to the health emergency, according to educational level, sex, age and academic training. We worked from a quantitative approach and a comparative descriptive design. The study sample consisted of 144 teachers from East Lima, to whom the questionnaire adapted and validated by Bolívar (2017): Evaluation of Professional Learning Communities, was applied virtually. The analysis carried out on the basis of the statistical data allowed to establish the differences at the level of the dimensions of the Professional Learning Communities; The research shows that the factors that involve the Professional Learning Communities in the Educational Institutions of East Lima, prevail a level of regular higher than $50 \%$, in the dimensions of shared leadership and support, shared vision and values, collective learning and application, shared professional practice, support conditions: relationships and support conditions: structure; followed by the efficient level; likewise, in all dimensions greater than $27.1 \%$; Therefore, it can be said that the professional communities under study have been adequately managed by the actors under study.

Keywords: professional learning communities, remote context, emergency 


\section{Introducción}

En el contexto actual, en el que sobrevino la emergencia sanitaria por la COVID-19 se presentó un escenario desafiante en todas las esferas de la sociedad, sobre todo en educación; debido a que enfrentó a toda la población educativa a un trabajo remoto en un panorama de incertidumbre en el que fue necesario poner en juego las capacidades y habilidades profesionales de los líderes educativos; pero no desde una perspectiva individual, sino colectiva, contando con la participación de todos los que conforman la Institución Educativa para dar continuidad al servicio educativo y de esta manera atender las necesidades de aprendizaje de los estudiantes. Malpica (2018) señala que, en la actualidad, se hace necesario trabajar en conjunto, enfocándose en los aprendizajes de los estudiantes; y más aún en este contexto remoto.

En ese sentido, las Comunidades Profesionales de Aprendizaje-CPA promueven el aprendizaje de los estudiantes a partir de estrategias pedagógicas validadas científicamente (Soria y Hernández, 2017). Por ello, desde las CPA se busca lograr una autonomía en el trabajo pedagógico a partir del protagonismo de los actores educativos con miras a alcanzar la sostenibilidad en las acciones implementadas (Bolívar, 2008), que en este contexto remoto se presentan como acciones desafiantes siendo fundamental promover y realizar acciones que contribuyan a un trabajo colaborativo eficiente.

Las CPA es una forma de organización muy poderosa para aprender en forma colaborativa y mejorar la práctica pedagógica del docente lo que permite mejores logros de aprendizaje, aun teniendo en cuenta que el término de comunidad que forma parte de la denominación de las CPA, surge de las prácticas de trabajo colaborativo que deja de lado el trabajo individualista y aislado que no ayudan a afrontar los desafíos que se presentan en el ámbito educativo (Bolívar, 2017). Este trabajo en conjunto logra transformar la organización de la Institución Educativa funcionando como un sistema coherente que apunta a un propósito claro y objetivos bien definidos (Fullan \& Quinn, 2017).

Asimismo, Pozner (1998) citado por Álvarez y Casas (2008) señala que a partir de la gestión escolar se debe establecer una coherencia del trabajo educativo en concordancia con los cambios que suceden en el mundo, considerándose estos en los documentos de gestión. En ese 
sentido, muchas veces se ha criticado que la educación no se desarrolla acorde con las exigencias del mundo actual; y por ello significó un impacto a todo nivel, este nuevo escenario, en el sector educación; puesto que no se estaba preparado para enfrentar una modalidad remota y mucho menos con el uso de recursos tecnológicos y virtuales. Teniendo en cuenta que este nuevo escenario no hizo distinción entre los docentes noveles con aquellos que están por cesar, la emergencia sanitaria hizo que dejaran la modalidad presencial en el que las estrategias y los recursos y materiales educativos eran otros (Arriagada, 2020); por ello, cobra sentido la coherencia sistémica que debe existir en la implementación de las CPA con relación a las exigencias del mundo actual.

Un aspecto muy importante que se debe considerar en toda Institución Educativa es el propósito que guíen todas las acciones de la organización escolar y por ello el papel del líder pedagógico juega un rol fundamental para promover las CPA en un escenario de trabajo remoto, como señala Rivero et al. (2018) una de las funciones de todo líder es guiar a toda su comunidad educativa a base de una visión y un propósito que deben surgir a partir de la participación y aporte de todos, los cuales guíen las acciones pedagógicas de soporte, de convivencia, entre otros hacia el logro de metas comunes que favorezcan la mejora de los aprendizajes.

En cuanto a los estudios realizados tenemos el de Morales \& Morales (2019) que realizaron una síntesis de las CPA basándose en cinco dimensiones en el que presentaron a la visión y valores compartidos como dos dimensiones distintas y no consideran la dimensión de aprendizaje colectivo y aplicación; es posible que hayan considerado como un aspecto implícito a partir de las otras dimensiones consideradas; puesto que más adelante señalan que es importante que los docentes aprendan nuevas formas de enseñar y que reflexionen de su práctica. Por otro lado, Sánchez (2016) refiere que existe una relación entre las dimensiones de las CPA y las prácticas de enseñanza de los docentes; sin embargo, la relación es débil en todos los casos; también, señala que los docentes que tienen mayor edad y más años de experiencia muestran mayor disposición para participar en actividades de comunidades profesionales de aprendizaje. Mellado et al. (2020) refieren en su estudio hallazgos referidos a las redes de líderes educadores y su relación estrecha con la participación democrática en función a proyectos y metas compartidas; asimismo, consideran la importancia de la participación de un acompañante externo que cumpla un papel crítico y de modelamiento en la ejecución de experiencias de liderazgo; hallazgos que contribuyen a la transformación de las redes en CPA. García-Martínez et al. 
(2018) concluyen que el liderazgo descentralizado es un componente primordial para propiciar el progreso en el ámbito educativo contribuyendo a su transformación en una CPA con la participación de todos, especialmente de los docentes; asimismo, aseveran que esta transformación se da en contextos desafiantes convirtiendo estos en oportunidades para el trabajo colaborativo de los docentes y se logren aprendizajes profesionales; es importante considerar este último hallazgo, puesto que en el contexto actual de inseguridad por la emergencia sanitaria se debe asumir como una oportunidad para constituir las CPA en todas sus dimensiones y posibilidades. Otro aspecto abordado en las CPA es la innovación pedagógica reflexiva, relacionadas con las dimensiones: aprendizaje colectivo y aplicación y práctica profesional compartida, en un estudio realizado por Malpica \& Navareño (2018) concluyendo que la innovación pedagógica reflexiva por medio de las comunidades profesionales de aprendizaje tendrá una atribución cuando exista un sistema de desarrollo profesional individual y colectivo de los docentes dentro de un proceso que considere la innovación, la flexibilidad y el dinamismo. Por último, un estudio realizado por Domingo-Segovia et al. (2020) presentan un cuestionario adaptado y validado del PLCA-R: Evaluación de Comunidades Profesionales de Aprendizaje para el habla hispana en el que consideran cinco dimensiones y 52 ítems, que permitirá evaluar o diagnosticar si una Institución Educativa es una comunidad profesional de aprendizaje.

Se define a las comunidades profesionales de aprendizaje como una forma de organización de las escuelas donde se prioriza la discusión y la reflexión sobre las nuevas pedagogías para mejorar el aprendizaje de los estudiantes (Krichesky, 2011), donde lo fundamental es la indagación y el aprendizaje de los docentes por lo que se puede considerar su implicancia en la mejora de la Institución Educativa (Hord \& Hirsh, 2008). Son consideradas también una estrategia de formación docente que permite el fortalecimiento de su práctica profesional (García \& Trigueros, 2015); sin embargo, Krichesky \& Murillo (2011) aseveran que las CPA son más que una estrategia, pues no se limita a un solo nivel educativo, o un grupo de profesionales dentro del espacio educativo, sino que esta puede constituirse en un enfoque de trabajo a nivel de toda la organización de la Institución Educativa.

En cuanto a su importancia las CPA son relevantes en la organización de las Instituciones Educativas; puesto que, tienen al aprendizaje de los estudiantes como el núcleo fundamental del trabajo colaborativo; es decir, el currículo de la educación (Bolívar, 2008). Por tanto, son inclusivas porque tienen la firme convicción de que todos los estudiantes pueden aprender 
(Escudero, 2009). Además, tiene como característica central el trabajo colaborativo incorporando las normas y valores compartidos, liderazgo distribuido y de apoyo, participación, clima de confianza y respeto; así como el diálogo reflexivo, una práctica compartida, aprendizajes individuales y colectivos y responsabilidad sobre los aprendizajes en forma conjunta (Krichesky, 2011). Por ello contribuye en tres aspectos: el crecimiento profesional del docente, la forma de cómo aprender y el progreso de los aprendizajes (Bond, 2013).

Se considera que para conformar una CPA se debe primero rediseñar los espacios y segundo desaprender la cultura instalada del individualismo para que poco a poco se pueda ir promoviendo una cultura de colaboración y en ello, puede contribuir como parte inicial del proceso, el desarrollo de proyectos de aprendizaje (Bolívar, 2008). Vivir los principios del aprendizaje dialógico son acciones muy potentes para promover y constituir una comunidad de aprendizaje, es decir, cuando todos tienen la oportunidad de hablar en forma horizontal va incrementando la participación de todos los actores educativos (Flecha \& Puigvert, 2002). Otra propuesta para desarrollar una CPA está referida a desarrollar las capacidades del docente como: capacidad de mantener diálogos sinceros, capacidad de gestionar conocimiento, la capacidad de relacionar su propio aprendizaje con su práctica pedagógica y lo que aprenden los estudiantes, la capacidad de ser abierto y compartir su práctica y la capacidad de desarrollar su liderazgo (Lieberman \& Miller, 2007, citado por Krichesky, 2013).

Asimismo, las comunidades de aprendizaje no pueden subsistir como islas tienen que establecer redes con otras Instituciones Educativas y aliados que contribuyan a su desarrollo y fortalecimiento, esto da sostenibilidad a las comunidades de aprendizaje porque aprenden unos de otros a partir de equipos de liderazgo que se reúnen periódicamente (Fullan \& Quinn, 2017). Conformar redes de aprendizaje tanto internas como externas es parte inherente de una CPA en el que se complementan con aquello que puede carecer una de la otra o compartir sus experiencias siendo una estrategia para la institucionalización de las CPA (Murillo, 2012); establecer redes tanto internas como externas en una modalidad remota es más factible que la modalidad presencial, puesto que, la barrera del tiempo y del espacio se ve superada porque no están sujetos a horarios limitados dentro del espacio escolar, ni tienen que movilizarse de un lugar a otro para reunirse; en consecuencia, existe más posibilidad de promover el trabajo colaborativo en forma virtual a partir de un horario consensuado; permitiendo enfrentar mejor los complejos desafíos del sistema educativo, especialmente en el contexto remoto. 
Las dimensiones de las CPA que se han considerado en este estudio son: liderazgo compartido y de apoyo, visión y valores compartidos, aprendizaje colectivo y aplicación, práctica profesional compartida, condiciones de apoyo-relaciones, condiciones de apoyo-estructura (Bolívar, 2017).

Liderazgo compartido y de apoyo, la Institución Educativa es un espacio de oportunidad de aprendizaje en el que todos los profesionales docentes, auxiliares, psicólogos, apoyos educativos, entre otros, en una CPA desarrollan su liderazgo a partir de su crecimiento profesional y se aceptan como personas que aprenden (Bolívar, 2017). Consiste en compartir el poder entre directivos y docentes para tomar decisiones sobre todo en aspectos relacionados con el currículo y el cambio de roles de los docentes (Hord, 1997). Una CPA no es sostenible si no cuenta con esta dimensión, ya que contribuye a la participación de todos los actores, a partir de su liderazgo, a afrontar en conjunto los desafíos de la organización escolar (Bolívar, 2017), ya que es tan compleja y recargada a nivel administrativo. La misma autora afirma que para desarrollar esta dimensión en una CPA es necesario que en la Institución Educativa se haya desarrollado cierta cultura de trabajo colaborativo para que pueda funcionar. Para Elmore (2010) el liderazgo implica el desarrollo de una coherencia organizacional que se conduce relación con el bien común, que se guía de unos protocolos que le permitan observar y analizar la práctica pedagógica.

Visión y valores compartidos, para desarrollarse como una CPA es esencial contar con una visión y valores que se plasman con los aportes de todos los actores de la institución escolar, esto permite que todos tengan una guía que dirija acciones basándose en este propósito compartido (Bolívar, 2017). El propósito de la CPA es centrarse en lo que aprenden los estudiantes (Hord, 1997). La visión muestra a dónde se quiere llegar y cómo seremos cuando se llegue allí, es el impulso que da sentido a las acciones que se realizan en una CPA (Rivero et al., 2018).

Aprendizaje colectivo y aplicación, se refiere a la indagación y reflexión sobre las estrategias, metodologías y toda investigación referente a adecuar el currículo de la educación a las necesidades y características de aprendizaje de los estudiantes en concordancia con la visión compartida y lo aplican (Bolívar, 2017). Este aprendizaje colectivo debe desarrollarse sobre la base de un diálogo igualitario en donde lo que se prioriza es la validez de los argumentos más que 
los cargos jerárquicos (Flecha \& Puigvert, 2002). Esto implica un nuevo docente con nuevas características que responda a las exigencias del mundo actual (Jimenez \& Gaitán, 2017).

Práctica profesional compartida, en una CPA, especialmente los directivos y los docentes se unen para analizar, reflexionar y mejorar la práctica docente (Bolívar, 2017). Los actores educativos profesionales comparten en forma recíproca sus conocimientos por el bien de su propio desarrollo profesional (Hord, 1997); y por ende el desarrollo del capital social repercute en el desarrollo institucional. La reflexión debe partir de preguntas referidas a qué deben aprender los estudiantes, cómo sabemos que han aprendido y qué solución vamos a dar cuando un estudiante presenta dificultades para aprender (DuFour, 2006).

Condiciones de apoyo-relaciones, concierne a las actitudes de respeto, sinceridad y amabilidad que deben existir entre los miembros de una CPA; así como la importancia de motivar y celebrar el logro individual y colectivo, esto les genera compromiso (Hord, 1997). Asimismo, se debe considerar la crítica y la mejora entre docentes, directivos y estudiantes (Bolívar, 2017).

Por último, las condiciones de apoyo-estructura, se refiere al espacio que propicie un encuentro colaborativo entre docentes y directivos; así como al horario que favorezca la participación colaborativa, que propicie el diálogo reflexivo sobre asuntos relacionados con la práctica y el aprendizaje de los estudiantes (Hord, 1997). Incluye, también, las formas de comunicación y el tamaño de la Institución Educativa (Bolívar, 2017).

Si bien es cierto actualmente se cuenta con muchos estudios sobre las CPA; sin embargo, es poco frecuente escuchar hablar de ello en las Instituciones Educativas de Lima Este y mucho menos realizar acciones focalizadas y sistemáticas que permitan constituirse como CPA. Por ello, se considera importante determinar las diferencias que existen en los factores que describen las Comunidades Profesionales de Aprendizaje en las Instituciones Educativas de Lima Este en un contexto de trabajo remoto por la emergencia sanitaria, según nivel educativo, sexo, edad y formación académica; debido a que no se han realizado estudios sobre esta variable en un contexto atípico por la Covid-19. Asimismo, su divulgación permitirá que se promuevan acciones de mejora en las Instituciones Educativas de Lima Este que deseen constituirse en una CPA, cuyas dimensiones se encuentren en un nivel de eficiencia bajo con respecto a otras. 


\section{Método}

El estudio realizado responde a un enfoque cuantitativo de diseño no experimental de tipo descriptivo, de nivel comparativo, de corte transversal en el que se realizó una descripción comparativa de los resultados obtenidos de la variable estudiada, según sus dimensiones. Este análisis fue objetivo basándose en la estadística, usando la recolección de datos para probar la hipótesis planteada.

La población estuvo representada por docentes de las Instituciones de Lima Este, siendo la muestra 144 docentes que por razones éticas se mantendrá sus nombres en reserva.

La técnica que se utilizó fue la encuesta y el instrumento fue el cuestionario adaptado y validado por Bolívar (2017) cuyo título es Evaluación de las Comunidades Profesionales de Aprendizaje; y que fue aplicado en forma virtual. El instrumento presentó como alternativas la escala de Likert (Totalmente en desacuerdo, en desacuerdo, de acuerdo, totalmente de acuerdo) con el que se recogió información respecto a la variable de estudio considerando sus seis dimensiones, con un total de 46 ítems sin considerar los ítems complementarios. Instrumento validado a partir de un análisis factorial exploratorio alcanzando un coeficiente de 0,974 sometiéndose a revalidación a través de un análisis factorial confirmatorio presentando un valor de 0,062, inferior a 1, en cuanto a la fiabilidad se utilizó el coeficiente Alfa de Cronbach arrojando un resultado de 0,963 considerado en nivel de fiabilidad del instrumento como buena o alta (Bolívar, 2017).

El análisis estadístico que se utilizó para el presente estudio fue el SPSS V.25, en el que se analizó los datos en forma descriptiva e inferencial tomando en cuenta los niveles de medición de las variables, según nivel educativo, sexo, edad y formación académica. 


\section{Resultados}

\section{Análisis descriptivo}

Como se observa en la tabla 1 y figura 1, las Comunidades Profesionales de Aprendizaje en las Instituciones Educativas de Lima Este en un contexto de trabajo remoto por la emergencia sanitaria, en un nivel regular representa más del 50\% (56.3\%), seguida de un nivel eficiente en un $29.9 \%$ e ineficiente en un $13.9 \%$; todo ello en una realidad de emergencia sanitaria.

\section{Tabla 1}

Comunidades Profesionales de Aprendizaje en las Instituciones Educativas de Lima Este.

\begin{tabular}{lcc}
\hline Nivel & Frecuencia & Porcentaje \\
\hline Ineficiente & 20 & 13,9 \\
Regular & 81 & 56,3 \\
Eficiente & 43 & 29,9 \\
Total & 144 & 100,0
\end{tabular}

Fuente: Base de datos cuestionario de Comunidades Profesionales de Aprendizaje y SPSS V.25

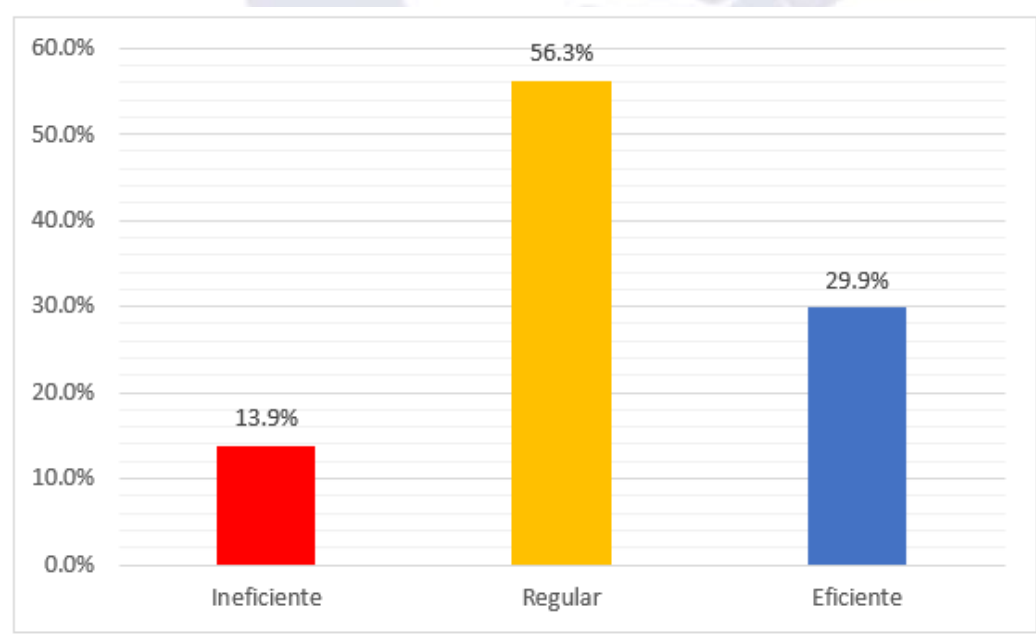

Figura 1: Diagrama de frecuencia de las Comunidades Profesionales de Aprendizaje en las Instituciones Educativas de Lima Este. 
Como se observa en la figura 2, los factores que involucran las Comunidades Profesionales de Aprendizaje en las Instituciones Educativas de Lima Este, prevalecen un nivel de regular superior al 50\%, en las dimensiones de liderazgo compartido y de apoyo, visión y valores compartidos, aprendizaje colectivo y aplicación, práctica profesional compartida, condiciones de apoyo-relaciones y condiciones de apoyo-estructura; seguido del nivel eficiente, igualmente en todas las dimensiones superiores al $27.1 \%$; por lo que se puede afirmar que las comunidades profesionales en estudio vienen gestionándose adecuadamente por parte de los propios actores en estudio.

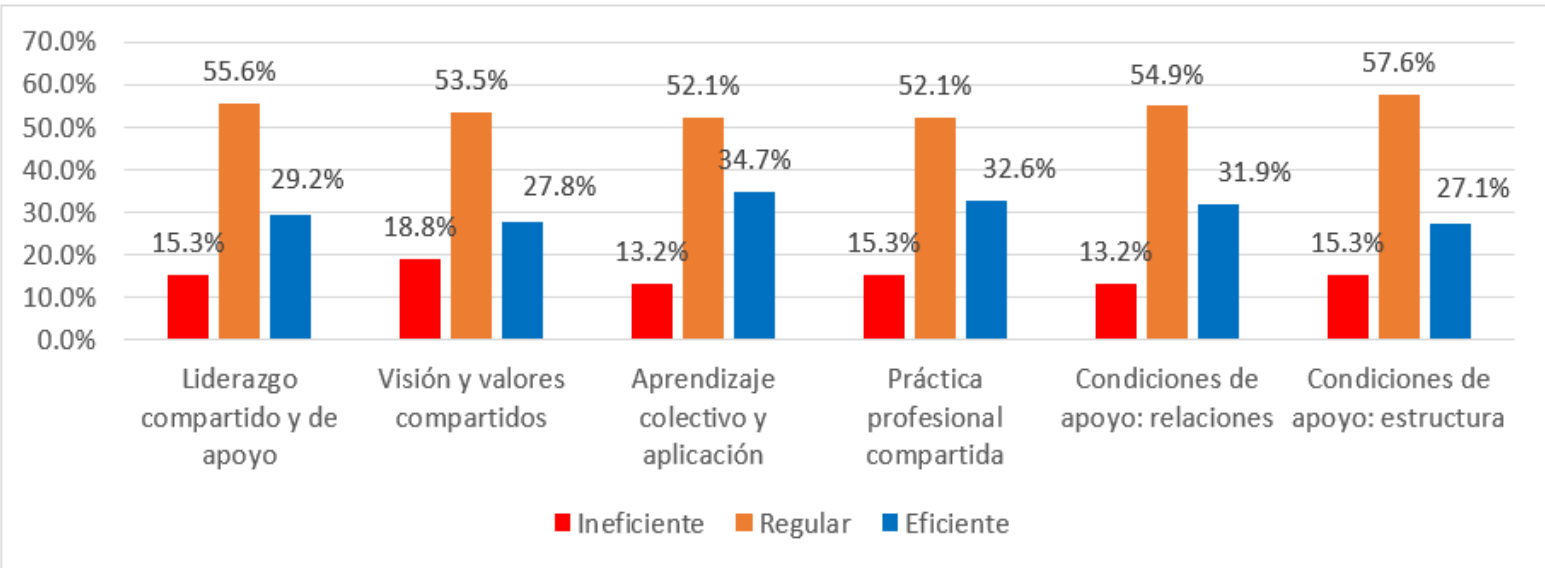

Figura 2: Diagrama de frecuencia de los factores que involucran las Comunidades Profesionales de Aprendizaje en las Instituciones Educativas de Lima Este.

Como se observa en la tabla 2, respecto a las Comunidades Profesionales de Aprendizaje en las Instituciones Educativas de Lima Este, se observa que prevalece un nivel educativo más eficiente en el nivel secundaria seguido de primaria y el inicial. 
Tabla 2

Comunidades Profesionales de Aprendizaje según nivel educativo

\begin{tabular}{llccc}
\hline & & \multicolumn{3}{c}{ Nivel Educativo } \\
\cline { 3 - 5 } & & & \multicolumn{2}{c}{ Secunda- } \\
& & Inicial & Primaria & ria \\
\hline Comunidades & Inefi- & 3 & 12 & 5 \\
profesionales & ciente & $37,5 \%$ & $18,8 \%$ & $6,9 \%$ \\
de aprendizaje & Regular & 5 & 39 & 37 \\
& & $62,5 \%$ & $60,9 \%$ & $51,4 \%$ \\
& & 0 & 13 & 30 \\
Total & & $0,0 \%$ & $20,3 \%$ & $41,7 \%$ \\
& & 8 & 64 & 72 \\
& & $100,0 \%$ & $100,0 \%$ & $100,0 \%$ \\
\hline
\end{tabular}

Fuente: Base de datos cuestionario de Comunidades Profesionales de Aprendizaje y SPSS V.25 Como se observa en la tabla 3, respecto a las Comunidades Profesionales de Aprendizaje en las Instituciones Educativas de Lima Este, prevalece un nivel educativo más eficiente en el grupo de 41- 50 años, seguidos de los grupos de 33,3\%, 51 - 60 años y 31 - 40 años.

\section{Tabla 3}

Comunidades Profesionales de Aprendizaje según nivel educativo grupo de edad

\begin{tabular}{lcccccc}
\hline & & \multicolumn{5}{c}{ Edad } \\
\cline { 3 - 7 } & & & & & 60 \\
& & & & & \\
& & años & años & años & más & 30 año \\
\hline Comunidades & Ineficiente & 7 & 7 & 6 & 0 & 0 \\
profesionales & & $28,0 \%$ & $15,6 \%$ & $9,7 \%$ & $0,0 \%$ & $0,0 \%$ \\
de aprendizaje & Regular & 15 & 19 & 37 & 8 & 2 \\
& & $60,0 \%$ & $42,2 \%$ & $59,7 \%$ & $88,9 \%$ & $66,7 \%$ \\
& & 3 & 19 & 19 & 1 & 1 \\
Eficiente & $12,0 \%$ & $42,2 \%$ & $30,6 \%$ & $11,1 \%$ & $33,3 \%$ \\
& & 25 & 45 & 62 & 9 & 3 \\
& & 100,0 & 100,0 & 100,0 & 100,0 & $100,0 \%$
\end{tabular}

Fuente: Base de datos cuestionario de Comunidades Profesionales de Aprendizaje y SPSS V.25 
Como se observa en la tabla 4, respecto a las Comunidades Profesionales de Aprendizaje en las Instituciones Educativas de Lima Este, se observa que presenta mejores niveles de eficiencia en los actores que tienen el grado de doctor seguidos los del maestría y licenciatura.

\section{Tabla 4}

Comunidades Profesionales de Aprendizaje según formación académica

\begin{tabular}{llccc}
\hline & & \multicolumn{3}{c}{ Formación académica } \\
\cline { 3 - 5 } & & \multicolumn{3}{c}{ Licen- } \\
& & Doctorado & ciado & Maestría \\
\cline { 2 - 5 } Comunidades & Ineficiente & 0 & 20 & 0 \\
profesionales & & $0,0 \%$ & $20,6 \%$ & $0,0 \%$ \\
de aprendizaje & Regular & 1 & 55 & 25 \\
& & $20,0 \%$ & $56,7 \%$ & $59,5 \%$ \\
& \multirow{2}{*}{ Eficiente } & 4 & 22 & 17 \\
& & $80,0 \%$ & $22,7 \%$ & $40,5 \%$ \\
& & 5 & 97 & 42 \\
& & $100,0 \%$ & $100,0 \%$ & $100,0 \%$ \\
\hline
\end{tabular}

Fuente: Base de datos cuestionario de Comunidades Profesionales de Aprendizaje y SPSS V.25

Como se observa en la tabla 5, respecto a las Comunidades Profesionales de Aprendizaje en las Instituciones Educativas de Lima Este, presenta mejores niveles de eficiencia en las mujeres que en los hombres. 
Tabla 5

Comunidades Profesionales de Aprendizaje según sexo

\begin{tabular}{llcc}
\hline & & \multicolumn{2}{c}{ Sexo } \\
\cline { 3 - 4 } & & Hombre & Mujer \\
\hline Comunida- & Inefi- & 3 & 17 \\
des profesio- & ciente & $7,5 \%$ & $16,3 \%$ \\
nales de & Regular & 27 & 54 \\
aprendizaje & & $67,5 \%$ & $51,9 \%$ \\
& Eficiente & 10 & 33 \\
& & $25,0 \%$ & $31,7 \%$ \\
Total & & 40 & 104 \\
& & $100,0 \%$ & $100,0 \%$
\end{tabular}

Fuente: Base de datos cuestionario de Comunidades Profesionales de Aprendizaje y SPSS V.25

\section{Análisis Inferencial}

\section{Prueba de Hipótesis}

Regla de decisión

Si Valor p > 0.05, se acepta la Hipótesis Nula (Ho)

Si Valor $\mathrm{p}<0.05$, se rechaza la Hipótesis Nula (Ho). Y, se acepta Ha

En la tabla 3, el resultado del p_valor de la prueba de Friedman p_valor $=0.003<0.05$; por tanto, se rechaza la Ho; aceptándose la hipótesis alterna de la variable. Por lo que se puede afirmar que existen diferencias significativas en los factores que describen las Comunidades

Profesionales de Aprendizaje en las Instituciones Educativas de Lima Este en un contexto de trabajo remoto por la emergencia sanitaria. 


\section{Tabla 3}

\section{Prueba de Friedman}

\begin{tabular}{lr}
\hline $\mathrm{N}$ & 144 \\
Chi-cuadrado & 17,728 \\
gl & 5 \\
Sig. asintótica &, 003 \\
\hline
\end{tabular}

a. Prueba de Friedman

Fuente: Base de datos cuestionario de Comunidades Profesionales de Aprendizaje y SPSS V.25

En la figura 3, se puede apreciar que la importancia que tiene cada factor respecto a las Comunidades Profesionales de Aprendizaje, siendo que el aprendizaje colectivo y la aplicación es la que tiene mayor importancia, seguido del resto de factores involucrados.

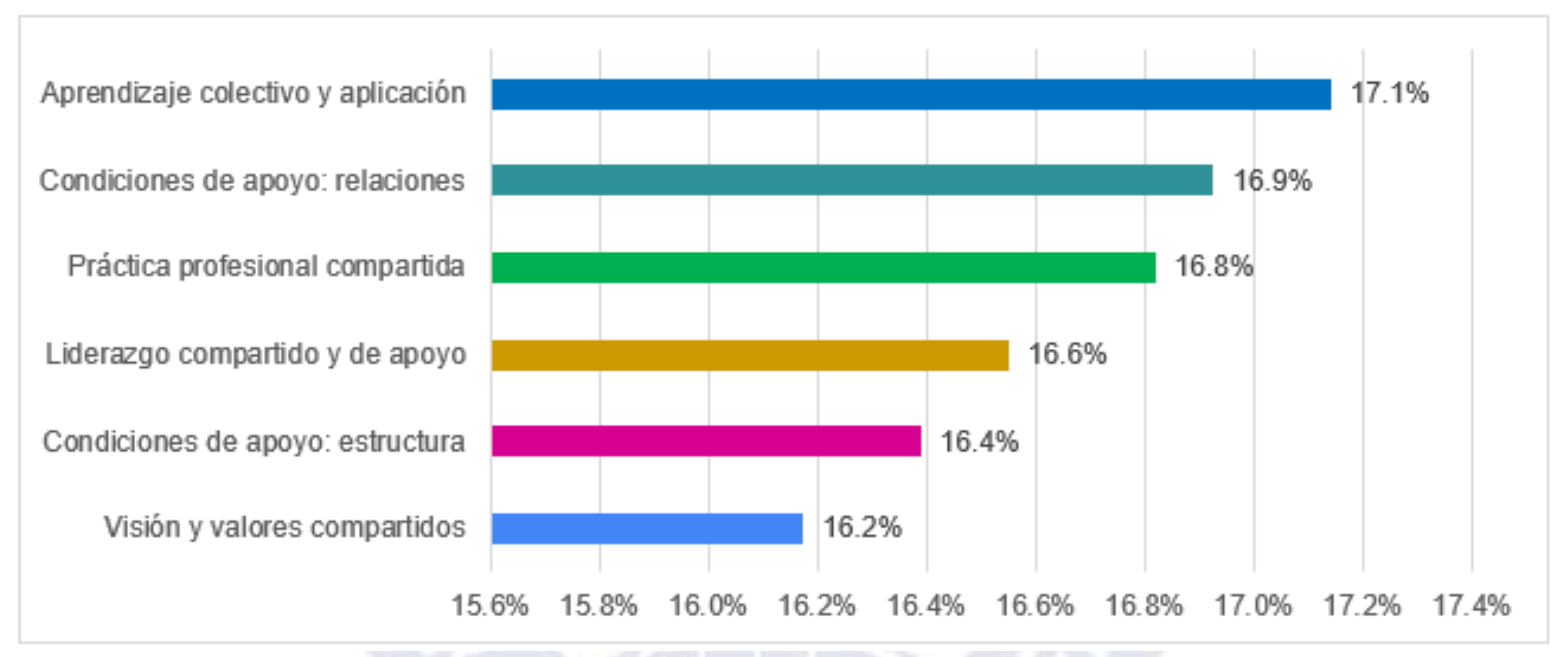

Figura 3: Diagrama de frecuencia de las Comunidades Profesionales de Aprendizaje según importancia de la eficiencia alcanzada

\section{Discusión}

La presente investigación respecto al objetivo general, se demuestra que el resultado del p_valor de la prueba de Friedman p_valor $=0.003<0.05$. Por lo que se puede afirmar que: existen diferencias significativas en los factores que describen las Comunidades Profesionales de Aprendizaje en las Instituciones Educativas de Lima Este en un contexto de trabajo remoto por 
la emergencia sanitaria; este resultado en el estudio realizado evidencia que cada dimensión o factor que involucrado en las CPA presenta situaciones diferenciadas en cuanto a cada actor involucrado en el estudio; siendo que el aprendizaje colectivo y aplicación resulta más relevantes para toda la muestra encuestada; ello puede deberse a que esta dimensión en el contexto actual por la emergencia sanitaria que se atraviesa evidencia la necesidad de entender otros enfoques educativos, técnicas y estrategias que permitan hacer más eficiente a estas comunidades profesionales para el logro de sus objetivos. Asimismo, se presenta el nivel más bajo en la dimensión o factor visión y valores compartidos lo que indica que las Instituciones Educativas de Lima Este requieren de una organización coherente que promueva en forma sistémica los componentes de una CPA; y esto se puede impulsar a partir del rol del líder pedagógico como lo asevera (Morales \& Morales, 2019) en un estudio realizado al respecto.

Igualmente, se puede evidenciar que en los factores que involucran las CPA en las Instituciones Educativas de Lima Este, prevalece un nivel de regular superior al 50\%, en las dimensiones de liderazgo compartido y de apoyo, visión y valores compartidos, aprendizaje colectivo y aplicación, práctica profesional compartida, condiciones de apoyo-relaciones y condiciones de apoyo-estructura; seguido del nivel eficiente que en todas las dimensiones fue superior al $27.1 \%$; por lo que se puede afirmar que las CPA en estudio vienen gestionándose adecuadamente por parte de sus propios actores y aquí se puede señalar que las circunstancias del trabajo remoto contribuyeron a acelerar el desarrollo individual y colectivo de los docentes tal como lo asevera Malpica \& Navareño (2018) que la reflexión pedagógica en una CPA se ve influenciada cuando existen estas condiciones de desarrollo profesional.

Se observa igualmente que las CPA en las Instituciones Educativas de Lima Este, prevalece un nivel educativo más eficiente en el nivel secundaria seguido de el de primaria y del nivel inicial, con una diferencia de $21.4 \%$ con respecto al nivel primaria y de $41.7 \%$ con respecto al nivel inicial como se aprecia en la Tabla 2; a partir de ello, se puede señalar que en el caso del nivel secundaria podrían haber condiciones que determinan el nivel más eficiente, debido a que en este nivel educativo se realizan reuniones de trabajo colegiado semanalmente establecidos por el Ministerio de Educación; otra condición, podría ser que este nivel educativo al ser atendido por varios docentes en un grado determinado, necesariamente implica abordar el currículo escolar en conjunto; una tercera condición podría ser que en este contexto de trabajo remoto los 
docentes del nivel secundaria han compartido espacios de enseñanza virtual que les permitió observarse entre pares.

Por otro lado, las CPA en las Instituciones Educativas de Lima Este, evidenció un nivel educativo más eficiente en el grupo de 41-50 años, seguidos de los grupos de 33,3\%, 51-60 años y 31-40 años; lo que corrobora el estudio realizado por Sánchez (2016). Se evidenció también que las CPA en las Instituciones Educativas de Lima Este, presentan mejores niveles de eficiencia en los actores que con grado de doctor, seguido por los de maestría y licenciatura, como lo referencia Sánchez (2016) en su estudio realizado en el que los niveles más altos fueron obtenidos por los docentes que tuvieron el grado de maestría; esto podría deberse a que en una CPA el docente que cuenta con mayor formación académica tiene mayores habilidades profesionales desarrolladas y por ello, más experiencia en el aprendizaje colaborativo y compartido que se promueven en estos espacios de formación profesional.

Por otro lado, las CPA en las Instituciones Educativas de Lima Este, se observó que presenta mejores niveles de eficiencia en las mujeres que en los hombres, presentando una diferencia de 6.7\%; sin embargo, los hombres presentan un nivel de ineficiente menor al de las mujeres, presentando una diferencia de $8.8 \%$. Otro resultado que se evidencia en la Tabla 5 , es referente a un porcentaje mayor de participación entre los encuestados; esto podría deberse a que en las Instituciones Educativas de Lima Este existen más docentes mujeres que hombres en los tres niveles educativos. Tal como lo corrobora el Instituto Nacional de Estadística e Informática (2017) en el que señala que el 63\% de docentes en el Perú son mujeres.

\section{Conclusiones}

1. La investigación evidencia que los factores que involucran las Comunidades Profesionales de Aprendizaje en las Instituciones Educativas de Lima Este, prevalecen un nivel de regular superior al 50\% en las dimensiones de liderazgo compartido y de apoyo, visión y valores compartidos, aprendizaje colectivo y aplicación, práctica profesional compartida, condiciones de apoyo-relaciones y condiciones de apoyo-estructura; seguido del nivel eficiente; igualmente, en todas las dimensiones superiores al 27.1\%; por lo que se puede afirmar que las comunidades profesionales en estudio vienen gestionándose adecuadamente por parte de los propios actores en estudio. 
2. El estudio realizado permitió demostrar que existen diferencias significativas en los factores que describen las CPA en las Instituciones Educativas de Lima Este en un contexto de trabajo remoto por la emergencia sanitaria, afirmando que el factor más alto es aprendizaje colectivo y aplicación con $17.1 \%$ y el nivel más bajo es visión y valores compartidos con 16.2, presentando una diferencia significativa de $0.9 \%$ entre ambos factores.

3. Se evidencia que en las CPA el nivel educativo más eficiente es el de secundaria con una diferencia de $21.4 \%$ con respecto al nivel que le precede que es el de primaria; asimismo se evidencia con respecto a la edad, que el rango con un nivel mayor de eficiencia es el de los docentes que cuentan entre 41 y 50 años de edad.

4. En las CPA la formación académica en el nivel más eficiente es alcanzado por aquellos docentes que tienen el grado académico de doctor con un $80 \%$ en los resultados estadísticos, seguido de los docentes que cuentan con maestría con un 40.5\%. Finalmente, en las CPA las mujeres presentan un nivel más eficiente con respecto a los hombres con una diferencia de $6.7 \%$.

\section{Referencias}

Álvarez, I., Casas M. (2008). Desafíos Para La Formación En Gestión. Experiencias Mexicanas. Investigación Administrativa, 37(102), 30-45. http://www.scielo.org.mx/pdf/ia/v37n102/2448-7678-ia-37-102-30.pdf

Arriagada, P. (2020). Pandemia Covid-19 : educación a distancia . O las distancias en la educación. Revista Internacional de Educación Para La Justicia Social, 9, 3. https://www.mendeley.com/catalogue/acff7184-2e8d-3afb-b6dd1453ada2cbf4/?utm_source=desktop\&utm_medium=1.19.4\&utm_campaign=open_catal og\&userDocumentId=\%7Bf74f19b7-f655-441f-9031-1e468bf37537\%7D

Bolívar, A. (2008). Otra alternativa de innovación, las comunidades profesionales de aprendizaje. October 2008, 2-18.

https://www.researchgate.net/publication/286060811_Otra_alternativa_de_innovacion_1 as_comunidades_profesionales_de_aprendizaje

Bolívar Ruano, M. R. (2017). Los centros escolares como comunidades profesionales de aprendizaje: Adaptación, validación y descripción del PLCA-R. 
http://hdl.handle.net/10481/47151

Bond, N. (2013). Developing a professional learning community among preservice teachers. Current Issues in Education, 16(2), 1-16.

Domingo-Segovia, J., Bolívar-Ruano, R., Rodríguez-Fernández, S., \& Bolívar, A. (2020). Professional Learning Community Assessment-Revised (PLCA-R) questionnaire: translation and validation in Spanish context. Learning Environments Research, 23(3), 347-367. https://doi.org/10.1007/s10984-020-09306-1

DuFour, R. (2004). What Is a “ Professional Learning Community "? Educational Leadership, 61(8), 6-11.

http://www.siprep.org/uploaded/ProfessionalDevelopment/Readings/PLC.pdf

Elmore, R. (2010). Mejorando la escuela desde la sala de clases. Santiago de Chile: Salesianos Impresores. www.fundacionchile.cl/educacion\%5CnInscripci $>n$

Escudero Muñoz, J. M. (2009). Comunidades Docentes De Aprendizaje, Formación Del Profesorado Y Mejora De La Educación. Ágora Para La Educación Física y El Deporte, 10, 7-31. http://uvadoc.uva.es/bitstream/10324/23683/1/AEFD-2009-10-comunidadesdocentes.pdf

Flecha García, J., \& Puigvert Mallart, L. (2002). La comunidades de aprendizaje: Una apuesta por la igualdad educativa. REXE: Revista de Estudios y Experiencias En Educación, 1(1), 11-20.

http://www.siprep.org/uploaded/ProfessionalDevelopment/Readings/PLC.pdf

Fullan M., Quinn J. (2017). Coherencia: Las fuerzas correctas en acción para escuelas, distritos y sistemas. Red Global de Aprendizaje. CEIBAL.

García-Martínez, I., Higueras-Rodríguez, L., \& Martínez-Valdivia, E. (2018). Hacia la Implantación de Comunidades Profesionales de Aprendizaje Mediante un Liderazgo Distribuido. Una Revisión Sistemática. REICE. Revista Iberoamericana Sobre Calidad, Eficacia y Cambio En Educación, 16(2), 117-132. https://doi.org/10.15366/reice2018.16.2.007

Hord, S. (1997). Professional learning communities: Communities of continuous inquiry and improvement. Leadership, 40(1), 58-59. https://files.eric.ed.gov/fulltext/ED410659.pdf Hord, S., Hirsh, S. (2008). Professional learning communities: Making the promise a reality. The Soul of Educational Leadership. pp. 23-40. https://books.google.es/books?hl=es\&lr=\&id=39yAwAAQBAJ\&oi=fnd\&pg=PT41\&dq=making + the + promise + a + reality $\&$ ots $=$ Gow $R r$ 
a1uJQ\&sig=2UkQ2bsjRfNzA3oePM7tThN0QbI\#v=onepage \&q=making\%20the $\% 20$ pro mise $\% 20 \mathrm{a} \% 20$ reality $\& \mathrm{f}=$ false

Instituto Nacional de Estadística e Informática. (2017). Nota de prensa. Nstituto Nacional de Estadística e Informática, 143, 1-2.

http://m.inei.gob.pe/media/MenuRecursivo/noticias/nota-de-prensa-n143-2017-inei.pdf

Jimenez Muñoz, Jorge Andrés; Rossi, Fernanda; Gaitán Riveros, C. (2017). La práctica

reflexiva como posibilidad de contrucción de saberes. Aportes a la formacion docente en Educación Física. Movimento, 23(2), 1-15.

https://www.redalyc.org/pdf/1153/115351637011.pdf

Krichesky, G. (2011). El desarrollo de las Comunidades Profesionales de Aprendizaje: procesos y factores de cambio para la mejora de las escuelas. REICE. Revista Iberoamericana Sobre Calidad, Eficacia y Cambio En Educación, 9(1), 373. repositorio.uam.es/xmlui/handle/10486/13311

Krichesky, G. J. (2013). E c p a . 1-373. https://repositorio.uam.es/bitstream/handle/10486/13311/62970_Krichesky Gabriela J.pdf

Krichesky, G., \& Murillo Torrecilla, F. (2011). Las comunidades profesionales de aprendizaje: una estrategia de mejora para una nueva concepción de escuela. REICE. Revista Iberoamericana Sobre Calidad, Eficacia y Cambio En Educación, 9(1), 65-83. https://www.redalyc.org/pdf/551/55118790005.pdf

Malpica Basurto, F. (2018). Comunidades Profesionales de Aprendizaje: ¿clave del cambio? Educacción, 1-5.

www.educaccionperu.orgdialogoinformadosobrepoliticaspublicashttps://www.educaccio nperu.org

Malpica Basurto, F., \& Navareño Pinadero, P. (2018). Innovación pedagógica reflexiva en comunidades profesionales de aprendizaje y su impacto en la formación docente institucional. In Innoeduca. International Journal of Technology and Educational Innovation (Vol. 4, Issue 1). https://doi.org/10.24310/innoeduca.2018.v4i1.4835

Mellado hernández, M. E., Rincón-Gallardo, S., Arevana Kenigs, O. A., \& Villagra Bravo, C. P. (2020). Acompanamiento a redes de lideres escolares para su transformacion en comunidades profesionales de aprendizaje. Perfiles Educativos, 42(169), 52-69. https://doi.org/10.22201/iisue.24486167e.2020.169.59363

Morales, S., \& Morales, O. (2019). Comunidades profesionales de aprendizaje : de la gestión 
empresarial a la gestión educativa. Revista Peruana de Investigación Educativa, February, 99-125. http://revistas.siep.org.pe/index.php/RPIE/art\%0Aicle/view/89

Murillo, F. J. (2012). El Proceso Del Cambio Escolar. Una Guía Para Impulsar Y Sostener La Mejora De Las Escuelas. REICE. Revista Iberoamericana Sobre Calidad, Eficacia y Cambio En Educación, 10(1), 26-43.

https://repositorio.uam.es/bitstream/handle/10486/660883/REICE_10_1_2.pdf?sequence $=1$

Rivera-García, J., \& Bernal-Trigueros, A. (2015). La intervención social universitaria: Proceedings T-VI. Ecorfan. https://www.ecorfan.org/proceedings/CDU_VI/TOMO_VI.pdf

Rivero, E., Zoro, B., \& Aziz, C. (2018). Construyendo una visión compartida para la educación del territorio. Liderez Educativos, 6, 2-22.

https://www.mendeley.com/catalogue/20be5a7d-624e-393e-a562-

624d30b77be7/?utm_source=desktop\&utm_medium=1.19.4\&utm_campaign=open_cata $\log \&$ userDocumentId=\%7Bd5dc3cb6-3511-4694-82d9-e985f7b71796\%7D

Sánchez Cortés, A. M. (2016). Innovación pedagógica desde la perspectiva de comunidades profesionales de aprendizaje. Revista Logos Ciencia \& Tecnología, 8(1), 1-10. https://doi.org/10.22335/rlct.v8i1.338

Soria, Maria Gabriela; Hernández Asensio, R. (2017). Aportes a la formación docente desde comunidades de aprendizaje. Revista Del Cisen Tramas/Maepova, 5(2), 131-145. http://ppct.caicyt.gov.ar/index.php/cisen/article/download/10864/10435 